\title{
Aprendizaje práctico de la medicina en entornos clínicos para alumnos de tercer curso. Consecución de objetivos de conocimiento
}

\author{
Pedro J. Romero-Palacios, M. Asunción Romero-López, Francisco J. Gómez-Jiménez, M. Teresa Miranda-León
}

Objetivo. Presentar los principales resultados obtenidos en la consecución de los objetivos de conocimiento en el proyecto denominado 'Iniciación clínica básica centrada en la práctica médica', una innovación docente desarrollada en la asignatura 'Patología general' de la licenciatura de medicina durante el curso académico 2009-2010.

Sujetos y métodos. Se seleccionaron 50 alumnos de tercero de medicina para el desarrollo del proyecto, cuya finalidad era contribuir a dotarles de los conocimientos y habilidades básicas para iniciarse en la atención sanitaria mediante la realización de prácticas clínicas orientadas y supervisadas en contextos asistenciales reales, con unos objetivos concretos y bajo la supervisión de médicos expertos.

Resultados. Los resultados obtenidos fueron excelentes, mostrando la adquisición de todos los objetivos propuestos. La gran mayoría de los alumnos consideraron muy positiva, a la par que enriquecedora, la experiencia.

Conclusión. La metodología propuesta, basada en las prácticas clínicas orientadas a la consecución de objetivos concretos, mejora el aprendizaje. La satisfacción tanto de los alumnos como de los profesores ha sido muy alta con los resultados obtenidos tras la implantación del proyecto.

Palabras clave. Autoevaluación. Competencias transversales. Estancias clínicas. Prácticas clínicas. Practicum. Supervisión.

Practical learning in clinical medicine for students in the third course of medicine degree.

Achievement of cognitive objectives

Aim. To present the main results obtained in the cognitive objectives in the project called 'Basic Clinical Initiation Centered Medical Practice', a teaching innovation developed in the introduction to Internal Medicine subject of medical degree during the academic year 2009-2010.

Subjects and methods. 50 students were selected in the third course of medicine degree for the development of the project, which aimed to equip them with basic knowledge and skills to start on health care by conducting guided and supervised clinical practice in real clinical settings, with specific targets and under the supervision of medical experts.

Results. The results were excellent, showing the purchase of all proposed objectives. Most students felt that it was a very positive and enriching experience.

Conclusion. The proposed methodology, based on the achievement of specific goals, contributes to improve learning. The satisfaction of both students and teachers has been very high with the results obtained after implementation of the project.

Key words. Clinical practice. Clinical stays. Practicum. Self-evaluation. Supervision. Transferable skills.

\section{Introducción}

La vertiente práctica en la enseñanza de la medicina es una necesidad permanente, que se ve reforzada por las nuevas tendencias y exigencias que suponen la integración de nuestra universidad en el Espacio Europeo de Educación Superior (EEES) [1]. En este sentido, en los programas de enseñanza de la medicina actuales, los contenidos prácticos adquieren un mayor peso específico [2].
La reforma curricular propuesta por la implantación del Marco Europeo de Educación Superior ha abierto un amplio debate en las facultades de medicina españolas sobre el objetivo fundamental del Grado en Medicina, llegándose al acuerdo de que se trata de 'formar profesionales competentes en el ámbito básico, con unas bases adecuadas para la posterior formación en cualquier especialidad médica y de acuerdo con las funciones que los médicos puedan ejercer en el sistema sanitario. Estas
Departamento de Medicina (P.J. Romero-Palacios, F.J. GómezJiménez); Departamento de Didáctica y Organización Escolar (M.A. Romero-López); Departamento de Estadística e Investigación Operativa (M.T. Miranda-León). Facultad de Medicina. Universidad de Granada. Granada, España.

Correspondencia:

Dr. Pedro J. Romero Palacios. Departamento de Medicina. Facultad de Medicina. Universidad de Granada. Avda. Madrid, 11. E-18012 Granada.

E-mail:

pjromero@ugr.es

Agradecimientos:

Al equipo decanal de la Facultad de Medicina por su continuo apoyo. A los profesionales que han participado como tutores clínicos en cada uno de los centros en los que se ha llevado a cabo este proyecto. A las direcciones de los centros hospitalarios que han acogido a nuestros alumnos, en especial al Dr. F. Martí, director médico de la Clínica de la Inmaculada de Granada, y al Dr. E. Fajardo, director médico del Hospital Nuestra Señora de la Salud de Granada.

Conflicto de intereses: No declarado.

Conflict of interests: None declared.

(c) 2012 Educación Médica 
competencias deberían incluir el conocimiento y la comprensión de las ciencias básicas, clínicas y sociales y del comportamiento, incluyendo la salud pública, la medicina comunitaria, y la ética médica relevantes para la práctica médica, las actitudes y habilidades clínicas (anamnesis, establecimiento del diagnóstico, procedimientos prácticos generales, habilidades de comunicación, tratamiento y prevención de la enfermedad, promoción de la salud, rehabilitación, razonamiento clínico y capacidad para resolver problemas) y la habilidad para llevar a cabo un aprendizaje durante toda la vida y un desarollo profesional continuo adecuado y en cualquier caso deben estar perfectamente interrelacionadas con la formación posgraduada', según la Sociedad Española de Educación Médica [3].

Una vez superado el periodo inicial de formación, desarrollado durante los dos primeros años de la licenciatura, y antes de pasar a los cursos de formación clínica, afrontando el trato directo con pacientes en el ámbito de los centros de atención sanitaria (hospitales, centros de salud, unidades de urgencias, etc.), el estudiante de medicina debe contar con la posibilidad de adquirir las competencias y capacidades necesarias para iniciarse en el ejercicio de la profesión médica a través de la realización de actividades prácticas que afiancen y contextualicen el aprendizaje de las materias a estudiar. Por otra parte, desde la publicación del informe del General Medical Council británico de 1993 [4], existe una fuerte corriente de opinión favorable a que los estudiantes tengan relación con la realidad asistencial en fases precoces de su formación [5].

La reforma curricular que afecta a los planes de estudio de la carrera de medicina sugiere que éstos se centren más en la adquisición de competencias que en la transmisión de información y de conocimientos teóricos, lo que influye indudablemente en el modo de entender la enseñanza y, sobre todo, en la metodología docente. Un factor clave para este objetivo es el contacto precoz del estudiante con la realidad médica y el trabajo en equipo interdisciplinar.

En este contexto, la finalidad de las prácticas sería enriquecer la formación básica, complementando los aprendizajes académicos (teóricos y prácticos) con la experiencia formativa, ligada a los aprendizajes que tienen lugar en los centros de trabajo [6]. Mediante la supervisión clínica, un profesor experto ofrece al estudiante su experiencia profesional, a la par que asesoramiento sobre un caso que está siendo objeto de atención profesional.

La actividad práctica que aquí se presenta pretende aproximar a los estudiantes a contextos profesionales reales, un espacio en el que se establece una relación dialéctica entre la teoría y la práctica que enriquece y favorece los aprendizajes, a la vez que dota de una visión real de la profesión, siendo el principio de la socialización profesional.

El proyecto de innovación docente (PID) denominado 'Iniciación clínica básica centrada en la práctica médica' se planteó como un complemento de la enseñanza teórica y práctica que reciben los estudiantes durante el tercer año de licenciatura, en la asignatura de 'Patología general', que es una asignatura troncal, anual, de 24 créditos, 10 de los cuales son prácticos. El objetivo fundamental se estableció en dotar a los alumnos de los conocimientos y habilidades básicas necesarias para iniciarse en la atención sanitaria, mediante la realización de prácticas clínicas orientadas y supervisadas en contextos asistenciales reales, con objetivos concretos y bajo la supervisión de médicos expertos. Por este motivo, se solicitó desde el Departamento de Medicina, y el Decanato de la facultad, a la Unidad de Innovación Docente de la Universidad de Granada el apoyo necesario para la realización de este proyecto.

Las actividades prácticas han tenido lugar en dos centros sanitarios granadinos y en cinco contextos asistenciales bien diferenciados y complementarios entre sí, como son: servicio de urgencias, planta de hospitalización, consultas externas, Unidad de Cuidados Intensivos (UCI) y quirófanos. Asimismo, también ha participado el Servicio de Emergencias Sanitarias (061).

Las estancias clínicas se realizaron en dos hospitales privados de Granada (Clínica Inmaculada Concepción y Hospital Nuestra Señora de la Salud), que tienen vigentes acuerdos de colaboración con la universidad, con el apoyo de la dirección de ambos centros, y con la colaboración voluntaria y desinteresada de un gran número de facultativos de todas las áreas asistenciales implicadas. En cada caso se asignó un profesor de la asignatura como coordinador docente, que compartía labores de coordinación con el director médico de cada centro. Asimismo, participó en el proyecto la Empresa Pública de Emergencias Sanitarias, mediante la realización de un seminario práctico en el que los estudiantes pudieron conocer en pequeños grupos la dotación, funcionamiento y protocolos de actuación de una UVI móvil.

\section{Descripción y objetivos del proyecto}

Con el PID 'Iniciación clínica básica centrada en la práctica médica' se ha pretendido facilitar a los estudiantes la adquisición de una serie de habilidades 
clínicas básicas para el ejercicio de la profesión. Los objetivos generales propuestos han sido:

- Conocer y desarrollar de forma práctica los apartados y estructura principal de una historia clínica.

- Conocer la importancia clínica y valoración de los síntomas más prevalentes en la patología médica (disnea, dolor torácico, dolor abdominal y déficit neurológico) y su importancia en los grandes síndromes, en el ámbito de medicina primaria y de urgencias.

- Conocer y aplicar de forma práctica los protocolos de actuación para la correcta valoración de estos síntomas y el síndrome en que están inmersos.

- Conocer y aplicar en casos concretos las guías clínicas de tratamiento de la disnea, dolor torácico, dolor abdominal y déficit neurológico.

- Conocer e indicar correctamente las exploraciones complementarias básicas para la valoración clínica de estos síntomas.

- Conocer y aplicar correctamente las normas básicas para acceder a una área quirúrgica.

- Conocer e indicar correctamente las exploraciones básicas previas a una intervención quirúrgica.

- Conocer los principios básicos de valoración inicial del paciente crítico, desde el punto de vista sindrómico.

- Conocer la existencia y estructura fundamental de la gestión por procesos.

- Conocer la existencia y disponibilidad de distintas fuentes documentales y de información solventes y de referencia: Cochrane, Medline, procesos asistenciales integrados, sociedades científicas...

\section{Metodología de la investigación evaluativa}

Como se refleja en la tabla I, los contenidos docentes propuestos en el PID completan el currículo del tercer curso de medicina, incorporando módulos de aprendizaje presenciales y no presenciales, mediante la realización de actividades dirigidas y supervisadas, autónomas o en grupo, y de prácticas clínicas en un contexto asistencial real, bajo la supervisión directa de médicos expertos.

Los estudiantes candidatos hicieron una primera preinscripción, de la que se seleccionaron los 50 participantes, en función de las calificaciones del primer examen parcial de la asignatura 'Patología general' y el expediente académico.

$\mathrm{Al}$ inicio y al final del PID se pidió a los estudiantes que respondieran a un cuestionario de autoevaluación (Tabla II), en el que se les preguntaba sobre el grado de conocimiento que tenían de distintos aspectos relacionados con los objetivos de conoci- miento del curso en cada una de las áreas específicas, y en otro apartado se pedía una autovaloración de las habilidades que cada uno poseía antes del inicio del curso. Igualmente, al finalizar el PID, se realizaron encuestas de satisfacción.

Para todos los ítems contenidos en los cuestionarios se han elaborado las correspondientes tablas de frecuencias absolutas y porcentajes, tanto al inicio como al final de la experiencia. Para analizar globalmente el cambio o mejora obtenida se han sumado las puntuaciones de cada bloque de preguntas (objetivos de conocimiento) en las distintas áreas, para cada estudiante participante en el proyecto.

Las puntuaciones medias iniciales y finales de cada uno de los bloques se han comparado mediante el test $t$ de Student para datos apareados, obteniéndose igualmente los correspondientes intervalos de confianza al 95\% para la media de las diferencias entre el inicio y el final. Dichas diferencias han resultado ser altamente significativas $(p<0,001)$ para todos los resultados comparados, obteniéndose siempre mayor puntuación al final que al inicio, lo que indica la efectividad del método de docencia aplicado.

\section{Resultados}

En la tabla II se resumen los datos correspondientes a las encuestas realizadas a los alumnos y relacionadas con la consecución de los objetivos generales de conocimiento. Asimismo, en las tablas III, IV, V, VI y VII se muestran los resultados obtenidos en los cuestionarios realizados a los alumnos antes y después de su paso por los servicios de urgencias, planta de hospitalización, consultas y cirugía.

En todos los casos se aprecian unas diferencias altamente significativas en las puntuaciones que se obtienen al inicio y al final de la participación del proyecto.

\section{Discusión}

En general, puede afirmarse que los estudiantes participantes consiguen adquirir o afianzar una serie de conocimientos básicos para la práctica clínica, como lo demuestran los cambios tan significativos que se constatan en los conocimientos relacionados con la recepción, valoración inicial y procedimiento de información a los pacientes, que se llevan a cabo en los distintos servicios. En este sentido se aprecian también cambios importantes en la adquisición de las habilidades necesarias para efectuar estas tareas de manera competente. 
Tabla I. Contenidos docentes de la asignatura de 'Patología general' y del proyecto de innovación docente.

\begin{tabular}{|c|c|c|c|}
\hline Actividad práctica & Módulo & Horas & Actividades formativas \\
\hline $\begin{array}{l}\text { Laboratorio de } \\
\text { habilidades clínicas }\end{array}$ & $\begin{array}{l}\text { Prácticas de } \\
\text { 'Patología general' }\end{array}$ & 15 & $\begin{array}{l}\text { Exploración física } \\
\text { Auscultación respiratoria } \\
\text { Auscultación cardiaca } \\
\text { Palpación abdominal } \\
\text { Técnicas específicas } \\
\text { Colocación de sondas: nasogástrica, uretral } \\
\text { Venopunción } \\
\text { Espirometrías } \\
\text { Ecografía } \\
\text { Tacto rectal } \\
\text { Interpretación de trazados electrocardiográficos } \\
\text { Maniobras de reanimación cardiopulmonar básica }\end{array}$ \\
\hline $\begin{array}{l}\text { Seminarios de } \\
\text { comunicación }\end{array}$ & $\begin{array}{l}\text { Prácticas de } \\
\text { 'Patología general' }\end{array}$ & 15 & Anamnesis clínica: sistemática de realización de una historia clínica \\
\hline Sesiones clínicas a & $\begin{array}{l}\text { Proyecto de } \\
\text { innovación }\end{array}$ & 12 & $\begin{array}{l}\text { Comentario de casos clínicos reales seleccionados por los propios estudiantes } \\
\text { Comentario de casos clínicos seleccionados por los profesores tutores }\end{array}$ \\
\hline Seminarios ${ }^{a}$ & $\begin{array}{l}\text { Proyecto de } \\
\text { innovación }\end{array}$ & 12 & $\begin{array}{l}\text { Interpretación de trazados electrocardiográficos } \\
\text { Lectura radiológica: radiografía de tórax posteroanterior y lateral y abdomen en vacío }\end{array}$ \\
\hline $\begin{array}{l}\text { Planta de } \\
\text { hospitalización }{ }^{b}\end{array}$ & $\begin{array}{l}\text { Proyecto de } \\
\text { innovación }\end{array}$ & 20 & $\begin{array}{l}\text { Atención supervisada a pacientes ingresados por: disnea, dolor torácico, dolor abdominal } \\
\text { y déficit neurológico } \\
\text { Elaboración de historias clínicas completas a pacientes ingresados por estos síntomas } \\
\text { Exploración física } \\
\text { Anamnesis sistemática }\end{array}$ \\
\hline $\begin{array}{l}\text { Consultas externas } \\
\text { hospitalarias }^{b}\end{array}$ & $\begin{array}{l}\text { Proyecto de } \\
\text { innovación }\end{array}$ & 20 & $\begin{array}{l}\text { Atención supervisada a pacientes que consultan por: disnea, dolor torácico, dolor } \\
\text { abdominal y déficit neurológico } \\
\text { Elaboración de historias clínicas completas a pacientes que consultan por estos síntomas } \\
\text { Exploración física } \\
\text { Anamnesis sistemática }\end{array}$ \\
\hline Servicio de urgencias ${ }^{b}$ & $\begin{array}{l}\text { Proyecto de } \\
\text { innovación }\end{array}$ & 20 & $\begin{array}{l}\text { Atención supervisada a pacientes que consultan por: disnea, dolor torácico, dolor abdominal } \\
\text { y déficit neurológico } \\
\text { Elaboración de historias clínicas completas a pacientes que consultan por estos síntomas } \\
\text { Exploración física } \\
\text { Anamnesis sistemática }\end{array}$ \\
\hline $\begin{array}{l}\text { Unidad de Cuidados } \\
\text { Intensivos }(\mathrm{UCI})^{\mathrm{b}}\end{array}$ & $\begin{array}{l}\text { Proyecto de } \\
\text { innovación }\end{array}$ & 20 & $\begin{array}{l}\text { Métodos de control y monitorización del paciente crítico } \\
\text { Ventilación mecánica invasiva y no invasiva } \\
\text { Cuidados de los pacientes en UCl } \\
\text { Protocolos de actuación ante parada cardiorrespiratoria }\end{array}$ \\
\hline Quirófanob & $\begin{array}{l}\text { Proyecto de } \\
\text { innovación }\end{array}$ & 20 & $\begin{array}{l}\text { Acceso a zonas quirúrgicas limpias } \\
\text { Valoración prequirúrgica de pacientes } \\
\text { Actuar como ayudante de equipo quirúrgico } \\
\text { Cuidados postoperatorios del paciente }\end{array}$ \\
\hline $\begin{array}{l}\text { Aprendizaje autónomo } \\
\text { dirigido, con apoyo de } \\
\text { la plataforma SWAD }\end{array}$ & $\begin{array}{l}\text { Proyecto de } \\
\text { innovación }\end{array}$ & 20 & $\begin{array}{l}\text { Estrategias de búsqueda de información clínica relevante } \\
\text { Identificación de los procesos asistenciales integrados relacionados con los síntomas } \\
\text { estudiados } \\
\text { Lectura de las guías rápidas de los procesos asistenciales } \\
\text { Búsqueda de información y lectura de guías de práctica clínica relacionadas con los } \\
\text { síntomas estudiados } \\
\text { Búsqueda de información en bases de datos médicas: Medline, Cochrane } \\
\text { Búsqueda de información clínica en páginas de sociedades científicas: Samfyc, SEPAR, } \\
\text { SEC, SEMI }\end{array}$ \\
\hline
\end{tabular}

Realización de informe final de autoevaluación

Realización de historias clínicas tipo. Comentario de historias clínicas propuestas por los profesores

${ }^{a}$ Actividades del programa de la asignatura; ${ }^{b}$ Actividades específicas del proyecto. SWAD: sistema web de apoyo a la docencia. 
Figura. Grado de consecución de los objetivos cognitivos generales.

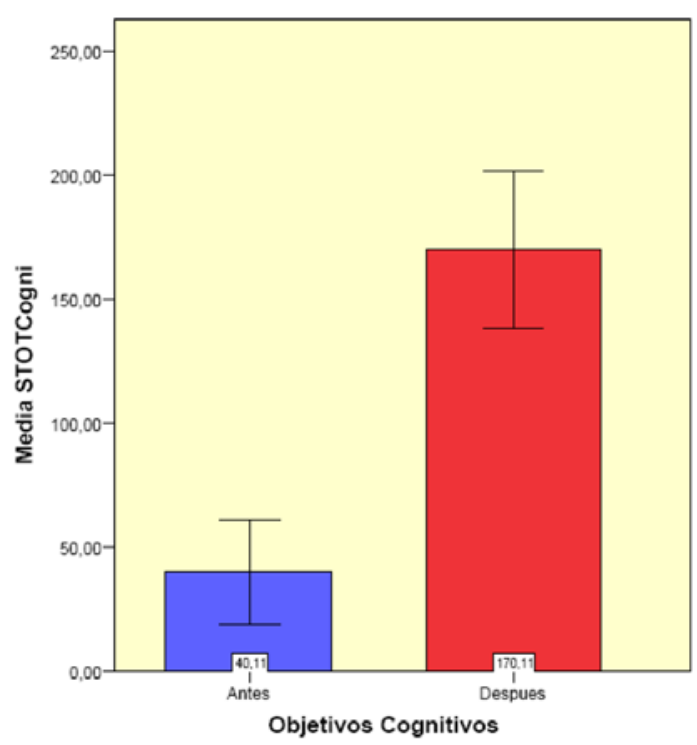

Por tanto, podemos confirmar, tras los resultados obtenidos, que los estudiantes terminan siendo capaces de reconocer clínicamente los síntomas objeto de estudio, así como de aplicar correctamente los criterios clínicos que definen la gravedad de éstos; ello pueden hacerlo en su mayoría de forma autónoma o bajo supervisión.

Los cambios más significativos se producen en los conocimientos y habilidades prácticas relacionadas con servicios y entornos clínicos en los que, por sus características específicas, los estudiantes han tenido menos experiencia previa. En este sentido es muy demostrativo el alto grado de satisfacción que manifiestan con respecto a las estancias en quirófanos y UCI, que son los servicios en los que se produce un mayor grado de progresión en lo referente a los objetivos de conocimiento que se proponían.

Los objetivos generales que se perseguían se han conseguido ampliamente. Si comparamos las puntuaciones totales del cuestionario referido a los objetivos generales antes y después de la realización del proyecto, la diferencia es altamente significativa (Figura). Puede apreciarse un cambio evidente en el conocimiento que tienen los estudiantes participantes acerca de todos los ítems, pero especialmente sobre el procedimiento de información a pacientes y familiares y la búsqueda de información. Los
Tabla II. Adquisición de objetivos generales de conocimiento.

\begin{tabular}{|c|c|c|c|}
\hline & & $\%$ inicial & $\%$ final \\
\hline \multirow{5}{*}{$\begin{array}{l}\text { Existencia de guías } \\
\text { de práctica clínica }\end{array}$} & No & 37,5 & 2,1 \\
\hline & Poco & 31,3 & 4,2 \\
\hline & Parcialmente & 20,8 & 25,0 \\
\hline & Suficientemente & 6,3 & 41,7 \\
\hline & En profundidad & 0 & 12,5 \\
\hline \multirow{5}{*}{$\begin{array}{l}\text { Existencia de procesos } \\
\text { asistenciales integrados }\end{array}$} & No & 47,9 & 0 \\
\hline & Poco & 35,4 & 8,3 \\
\hline & Parcialmente & 12,5 & 27,1 \\
\hline & Suficientemente & 0 & 39,6 \\
\hline & En profundidad & 0 & 10,4 \\
\hline \multirow{5}{*}{$\begin{array}{l}\text { Principales procesos } \\
\text { asistenciales integrados } \\
\text { relacionados con } \\
\text { los síntomas estudiados }\end{array}$} & No & 52,1 & 0 \\
\hline & Poco & 33,3 & 10,4 \\
\hline & Parcialmente & 10,4 & 29,2 \\
\hline & Suficientemente & 0 & 35,4 \\
\hline & En profundidad & 0 & 10,4 \\
\hline \multirow{5}{*}{$\begin{array}{l}\text { Fuentes de } \\
\text { información clínica }\end{array}$} & No & 20,8 & 0 \\
\hline & Poco & 56,3 & 8,3 \\
\hline & Parcialmente & 18,8 & 27,1 \\
\hline & Suficientemente & 0 & 37,5 \\
\hline & En profundidad & 0 & 12,5 \\
\hline \multirow{5}{*}{$\begin{array}{l}\text { Procedimiento } \\
\text { de información a } \\
\text { pacientes y familiares }\end{array}$} & No & 22,9 & 0 \\
\hline & Poco & 35,4 & 10,4 \\
\hline & Parcialmente & 29,2 & 16,7 \\
\hline & Suficientemente & 8,3 & 35,4 \\
\hline & En profundidad & 0 & 22,9 \\
\hline \multirow{5}{*}{$\begin{array}{l}\text { Búsqueda } \\
\text { de información }\end{array}$} & No & 16,7 & 0 \\
\hline & Poco & 33,3 & 8,3 \\
\hline & Parcialmente & 33,3 & 27,1 \\
\hline & Suficientemente & 12,5 & 27,1 \\
\hline & En profundidad & 0 & 22,9 \\
\hline
\end{tabular}

Las puntuaciones medias iniciales y finales se han comparado mediante el test $t$ de Student para datos apareados. Las diferencias han resultado ser altamente significativas $(p<0,001)$ para todos los resultados comparados. 
Tabla III. Objetivos de conocimiento en el servicio de urgencias.

\begin{tabular}{|c|c|c|c|c|c|}
\hline & $\%$ inicial & $\%$ final & & $\%$ inicial & $\%$ final \\
\hline $\begin{array}{l}\text { Documentos contenidos en } \\
\text { una historia clínica de urgencias }\end{array}$ & & & Criterios de ingreso y derivación & & \\
\hline No & 20,8 & 0 & No & 69,6 & 0 \\
\hline Poco & 47,8 & 0 & Poco & 23,9 & 2,4 \\
\hline Parcialmente & 20,8 & 0 & Parcialmente & 6,5 & 24,4 \\
\hline Suficientemente & 6,3 & 37,5 & Suficientemente & 0 & 48,8 \\
\hline En profundidad & 0 & 47,9 & En profundidad & 0 & 24,4 \\
\hline Recepción de pacientes & & & Cuidados del paciente en el área de urgencias & & \\
\hline No & 35,4 & 0 & No & 50,0 & 0 \\
\hline Poco & 33,3 & 0 & Poco & 45,7 & 0 \\
\hline Parcialmente & 20,8 & 4,2 & Parcialmente & 2,2 & 22,0 \\
\hline Suficientemente & 6,3 & 35,4 & Suficientemente & 2,2 & 41,5 \\
\hline En profundidad & 0 & 45,8 & En profundidad & 0 & 36,6 \\
\hline Valoración inicial del paciente & & & $\begin{array}{l}\text { Datos y constantes que se anotan } \\
\text { en historia clínica de urgencias }\end{array}$ & & \\
\hline No & 33,3 & 0 & No & 21,7 & 0 \\
\hline Poco & 35,4 & 0 & Poco & 50,0 & 0 \\
\hline Parcialmente & 20,8 & 6,3 & Parcialmente & 23,9 & 2,4 \\
\hline Suficientemente & 6,3 & 39,6 & Suficientemente & 0 & 43,9 \\
\hline En profundidad & 0 & 39,6 & En profundidad & 0 & 53,7 \\
\hline
\end{tabular}

\section{Métodos de priorización por gravedad}

No

Poco

Parcialmente

Suficientemente

En profundidad

Criterios clínicos para definir los síntomas

No

Poco

Parcialmente

Suficientemente

En profundidad

Criterios clínicos de gravedad

No

Poco

Parcialmente

Suficientemente

En profundidad

$\begin{array}{cc}41,3 & 0 \\ 37,5 & 4,9 \\ 15,2 & 14,6 \\ 6,5 & 41,5 \\ 0 & 39,0\end{array}$

39,0

$13,0 \quad 0$

$43,5 \quad 0$

$34,8 \quad 12,2$

$6,5 \quad 63,4$

$2,2 \quad 24,4$

$29,2 \quad 0$

$33,3 \quad 4,9$

$29,2 \quad 22,0$

$4,2 \quad 53,7$

$0 \quad 19,5$
Indicaciones de las exploraciones complementarias

$\begin{array}{lcc}\text { No } & 41,3 & 0 \\ \text { Poco } & 43,5 & 0 \\ \text { Parcialmente } & 15,2 & 9,8 \\ \text { Suficientemente } & 0 & 56,1 \\ \text { En profundidad } & 0 & 34,1\end{array}$

Criterios clínicos de situación de parada cardiorrespiratoria

No

Poco

$15,2 \quad 0$

Parcialmente

43,5 0

Suficientemente

$15,2 \quad 17,1$

En profundidad

$23,9 \quad 51,2$

$2,2 \quad 31,7$

Protocolo de actuación en parada cardiorrespiratoria

\begin{tabular}{lcc} 
No & 17,4 & 0 \\
Poco & 34,8 & 2,4 \\
Parcialmente & 21,7 & 19,5 \\
Suficientemente & 19,6 & 51,2 \\
En profundidad & 6,5 & 26,8 \\
\hline
\end{tabular}


Tabla IV. Objetivos de conocimiento en planta de hospitalización.

\begin{tabular}{|c|c|c|c|c|c|}
\hline & $\%$ inicial & $\%$ final & & $\%$ inicial & $\%$ final \\
\hline Documentos contenidos en una historia clínica & & & Cuidado de la vía venosa periférica & & \\
\hline No & 30,4 & 0 & No & 71,7 & 2,4 \\
\hline Poco & 34,8 & 0 & Poco & 26,1 & 2,4 \\
\hline Parcialmente & 21,7 & 0 & Parcialmente & 2,2 & 9,8 \\
\hline Suficientemente & 13,0 & 48,8 & Suficientemente & 0 & 48,8 \\
\hline En profundidad & 0 & 51,2 & En profundidad & 0 & 36,6 \\
\hline $\begin{array}{l}\text { Procedimientos de preparación } \\
\text { de la medicación diaria }\end{array}$ & & & Cuidado de la vía venosa central & & \\
\hline No & 67,4 & 0 & No & 76,1 & 2,4 \\
\hline Poco & 21,7 & 9,8 & Poco & 21,7 & 2,4 \\
\hline Parcialmente & 10,9 & 24,4 & Parcialmente & 2,2 & 14,6 \\
\hline Suficientemente & 0 & 48,8 & Suficientemente & 0 & 51,2 \\
\hline En profundidad & 0 & 17,1 & En profundidad & 0 & 29,3 \\
\hline Cuidados de los pacientes hospitalizados & & & Indicación de exploraciones complementarias & & \\
\hline No & 47,8 & 0 & No & 50 & 0 \\
\hline Poco & 37,0 & 2,4 & Poco & 39,1 & 0 \\
\hline Parcialmente & 15,2 & 26,8 & Parcialmente & 10,9 & 31,7 \\
\hline Suficientemente & 0 & 46,3 & Suficientemente & 0 & 48,8 \\
\hline En profundidad & 0 & 24,4 & En profundidad & 0 & 19,5 \\
\hline Anotación de constantes en la historia clínica & & & $\begin{array}{l}\text { Carro de parada: objetivos, componentes } \\
\text { y mantenimiento }\end{array}$ & & \\
\hline No & 37,0 & 0 & No & 69,6 & 2,5 \\
\hline Poco & 39,1 & 0 & Poco & 23,9 & 12,5 \\
\hline Parcialmente & 19,6 & 2,4 & Parcialmente & 4,3 & 12,5 \\
\hline Suficientemente & 4,3 & 58,5 & Suficientemente & 2,2 & 50 \\
\hline En profundidad & 0 & 39,0 & En profundidad & 0 & 22,5 \\
\hline
\end{tabular}

ítems que menos incidencia han recibido han sido el conocimiento de las fuentes relevantes de información clínica y los principales procesos asistenciales integrados relacionados con los síntomas estudiados. Ambos objetivos estaban planteados fundamentalmente mediante actividades de aprendizaje autónomo dirigido, lo que probablemente haya contribuido a que no se alcancen en su totalidad.

Son importantes los cambios que se aprecian en el conocimiento de los documentos que forman parte de una historia clínica, tanto en consulta como en planta de hospitalización o urgencias, lo cual constituía uno de los ejes centrales del proyecto. Es de destacar que gran parte de los alumnos fueron capaces de realizar historias clínicas completas a pacientes reales, en las que se recogía suficiente información como para tomar decisiones clínicas de manera fundamentada.

En el servicio de urgencias es destacable el cambio que se constata en el conocimiento de los documentos que forman parte de una historia clínica de urgencias y el modo en el que se recibe a los pacientes, así como la manera de evaluación inicial de éstos y los criterios por los cuales se establece la prioridad en la atención clínica, en función de la gravedad. Los objetivos que se alcanzan en menor proporción son el conocimiento de los criterios clínicos de gravedad y los criterios de ingreso y derivación. 
Tabla V. Objetivos de conocimiento en consulta.

\begin{tabular}{|c|c|c|c|c|c|}
\hline & $\%$ inicial & $\%$ final & & $\%$ inicial & $\%$ fina \\
\hline $\begin{array}{l}\text { Documentos contenidos en } \\
\text { una historia clínica de consulta }\end{array}$ & & & $\begin{array}{l}\text { Criterios clínicos necesarios } \\
\text { para definir los síntomas }\end{array}$ & & \\
\hline No & 17,4 & 2,4 & No & 17,4 & 0 \\
\hline Poco & 54,3 & 7,3 & Poco & 50,0 & 7,3 \\
\hline Parcialmente & 15,2 & 17,1 & Parcialmente & 30,4 & 19,5 \\
\hline Suficientemente & 13,0 & 53,7 & Suficientemente & 2,2 & 58,5 \\
\hline En profundidad & 0 & 19,5 & En profundidad & 0 & 14,6 \\
\hline Historia clínica básica & & & Criterios clínicos de gravedad de los síntomas & & \\
\hline No & 13,0 & 0 & No & 30,4 & 0 \\
\hline Poco & 41,3 & 4,9 & Poco & 52,2 & 12,2 \\
\hline Parcialmente & 30,4 & 22,0 & Parcialmente & 17,4 & 36,6 \\
\hline Suficientemente & 15,2 & 53,7 & Suficientemente & 0 & 36,6 \\
\hline En profundidad & 0 & 19,5 & En profundidad & 0 & 14,6 \\
\hline Pacientes en revisión y primera valoración & & & Criterios de ingreso y derivación & & \\
\hline No & 37 & 4,9 & No & 63,0 & 7,3 \\
\hline Poco & 32,6 & 7,3 & Poco & 34,8 & 7,3 \\
\hline Parcialmente & 21,7 & 34,1 & Parcialmente & 2,2 & 34,1 \\
\hline Suficientemente & 8,7 & 29,3 & Suficientemente & 0 & 31,7 \\
\hline En profundidad & 0 & 24,4 & En profundidad & 0 & 19,5 \\
\hline Priorización de exploraciones complementarias & & & Indicaciones de las exploraciones complementarias & & \\
\hline No & 50,0 & 7,3 & No & 39,1 & 4,9 \\
\hline Poco & 32,6 & 12,2 & Poco & 52,2 & 7,3 \\
\hline Parcialmente & 15,2 & 36,6 & Parcialmente & 8,7 & 31,7 \\
\hline Suficientemente & 2,2 & 29,3 & Suficientemente & 0 & 39,0 \\
\hline En profundidad & 0 & 14,6 & En profundidad & 0 & 17,1 \\
\hline
\end{tabular}

En este sentido, la correcta aplicación de dichos criterios requiere unos conocimientos que los alumnos de tercero de licenciatura aún no han tenido la oportunidad de estudiar.

En el servicio de planta de hospitalización también se ve notablemente incrementado el conocimiento acerca de los documentos contenidos en una historia clínica y la anotación de las constantes en ella. Los objetivos que se adquieren en menor proporción son el conocimiento de los procedimientos de preparación de la medicación diaria y la indicación de exploraciones complementarias.
En consulta se valora la adquisición del conocimiento sobre los pacientes en revisión y primera valoración, al igual que el conocimiento de los documentos contenidos en una historia clínica de consulta y el conocimiento de una historia clínica básica y los criterios de ingreso y derivación.

Son llamativos los resultados obtenidos en el área de cirugía y quirófano, pues son los que más cambio han experimentado, debido al desconocimiento inicial, mayor que el de las demás secciones o áreas. El cambio más importante se concentra en el conocimiento de lavado de manos y el acceso a la 
Tabla VI. Objetivos de conocimiento en servicio de cirugia y quirófano.

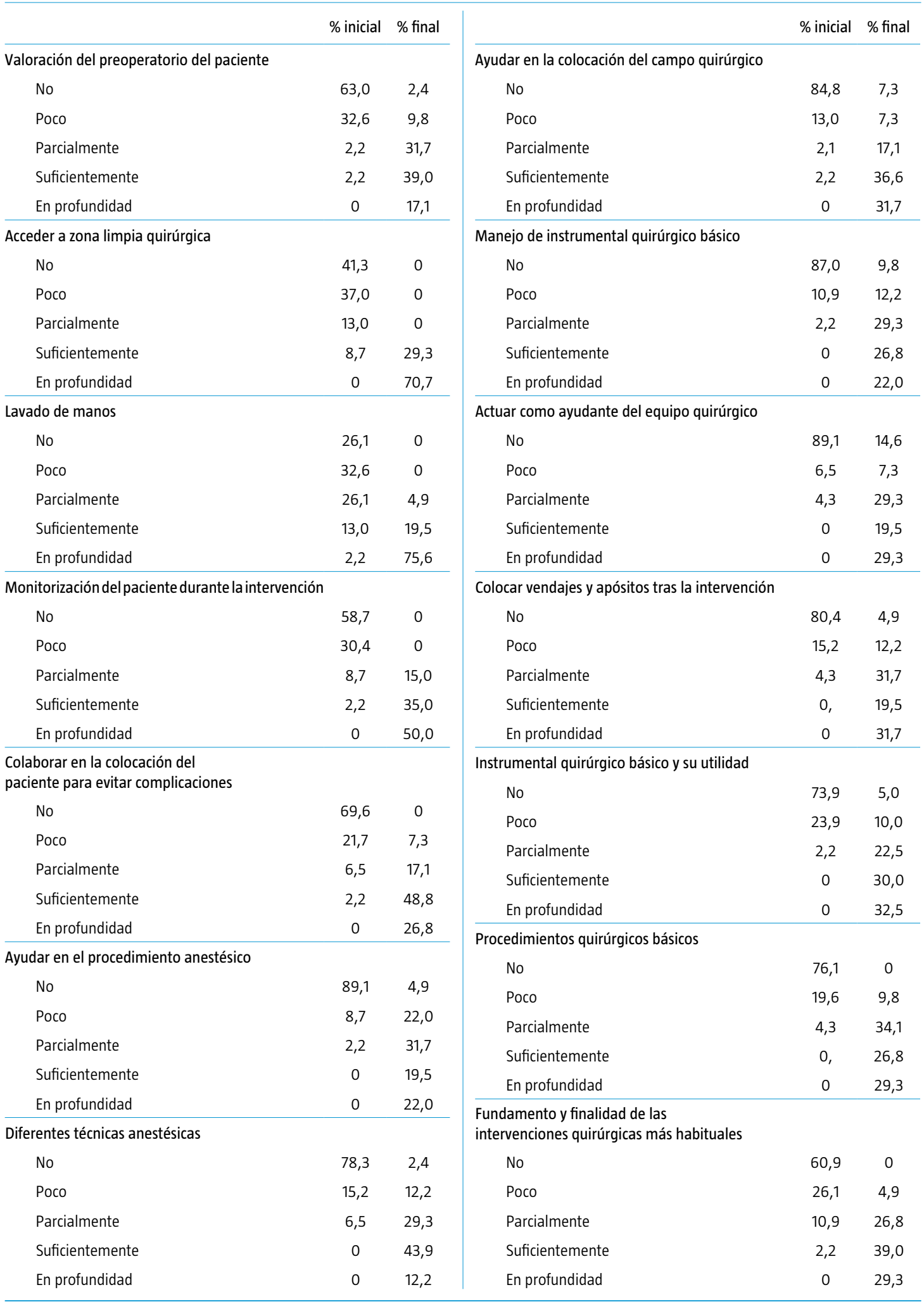


Tabla VII. Objetivos de conocimiento en la Unidad de Cuidados Intensivos (UCI).

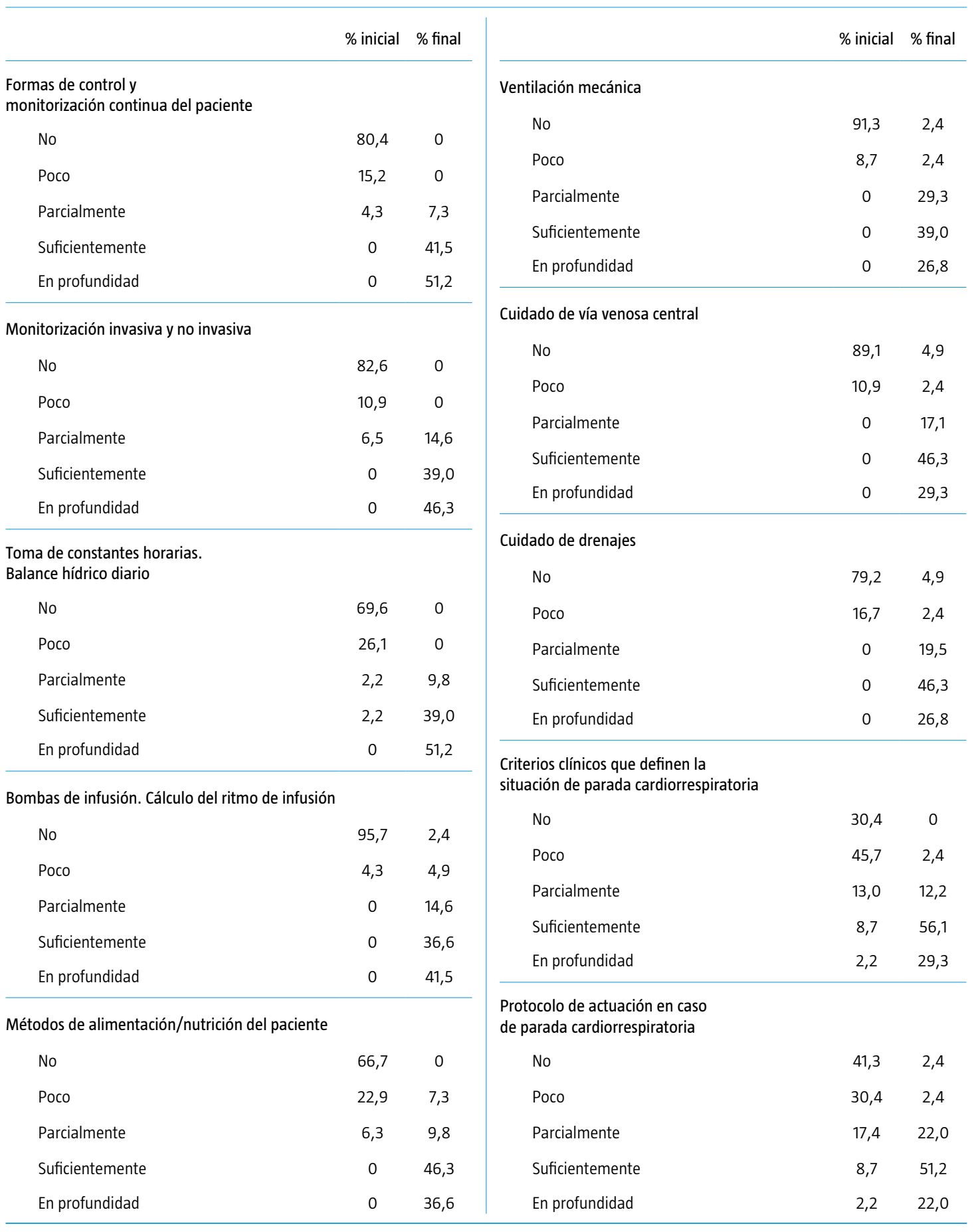

zona limpia quirúrgica. En el lado contrario encontramos ayudar en el procedimiento anestésico, el manejo de instrumental quirúrgico básico y actuar como ayudante de equipo quirúrgico.
En lo que respecta al servicio de cuidados intensivos, se adquiere en mayor proporción el conocimiento sobre las constantes horarias y balance hídrico y las formas de control y monitorización continua 
del paciente. En este sentido, destaca el alto grado de satisfacción de los estudiantes con las enseñanzas prácticas que reciben del personal de enfermería, en lo referente al manejo de dicha información en las hojas específicas para ello. Los conocimientos que se adquieren en menor proporción son los referentes a las distintas técnicas de ventilación mecánica, el cuidado de drenajes y el conocimiento del protocolo de actuación en parada cardiorrespiratoria. Como se ha comentado, se atribuye este hecho a que los alumnos de tercero de licenciatura carecen de la formación teórica necesaria para asimilar en profundidad estos conocimientos. No obstante, en la gran mayoría despiertan el interés y la necesidad de conocer más en profundidad estos aspectos de la atención médica, dotando de significado clínico directo materias que han de estudiar en cursos superiores.

El cumplimiento de los objetivos y el grado de satisfacción han sido realmente altos en todos los casos, a lo que probablemente haya contribuido el hecho de disponer de unos objetivos concretos a cumplir. En nuestra opinión, un aspecto clave ha sido que cada estudiante tuviese a su disposición un listado de objetivos a cumplir en cada estancia clínica, lo cual ha ayudado a centrar su atención en los aspectos concretos y en los objetivos a conseguir. Por su parte, los tutores clínicos también eran conocedores de estos objetivos, lo que les ha permitido ayudar a los estudiantes a conseguirlos.

Se ha demostrado que el curso (PID) ha servido para la adquisición, de forma estructurada, de los conocimientos y habilidades necesarias para hacer una valoración clínica inicial de los síndromes más prevalentes de la patología médica (disnea, dolor torácico, dolor abdominal y déficit neurológico), que constituyen la base de la mayor parte de los cuadros clínicos que motivan las consultas en la atención primaria y en los servicios de urgencias. La práctica clínica contribuye de manera decisiva a que los estudiantes adquieran competencias y habilidades que de otra forma no pueden llegar a adquirir, ya que es el contacto con la realidad médica y el trabajo en equipo lo que las potencia; ello coinciden con lo afirmado por otros autores en el sentido de que las prácticas profesionales son un espacio predilecto para el inicio a la socialización profesional [7-11].

En la formación de los futuros profesionales, la adquisición de competencias se convierte en un elemento clave para el éxito de la nueva forma de estudio. En este sentido, con el PID, se han utilizado diversas formas de evaluación de los conocimientos y competencias que adquieren los estudiantes, que a su vez han servido de incentivo para su consecución. La elaboración de historias clínicas centradas en pro- blemas ha sido un instrumento que se ha revelado especialmente útil al respecto, pues obliga al estudiante a concretar conocimientos y procesos de estudio de problemas clínicos reales. La supervisión continua de los profesores tutores supone un estímulo importante a la hora de conseguir estos objetivos, ayudando a aclarar conceptos y entender las actuaciones habituales en la práctica clínica [12].

Por otra parte, la posibilidad que se ofrece a los estudiantes de acceder a entornos clínicos asistenciales reales contribuye a que se hagan una idea clara de la realidad de la atención clínica. El acceso a planta de hospitalización, UCI y quirófano supone una oportunidad excelente para tomar contacto con aspectos concretos de la atención sanitaria que pueden resultar de gran interés para los estudiantes. La sistemática empleada para la toma de constantes en UCI, la forma segura de acceder a espacios quirúrgicos y el cuidado de vías centrales o sondas de drenaje constituyen aspectos de la atención sanitaria que los estudiantes absorben con auténtico interés y que les ayudan a conocer el medio y a situarse en la realidad clínica. En este sentido hay que valorar lo aportado por enfermería, como elemento importante en todo el conjunto de actividades que los estudiantes han llevado a cabo.

Como instrumentos de evaluación se ha demostrado que se han de potenciar aquellos que se ajustan mejor a la evaluación de habilidades y actitudes, como los circuitos de evaluación clínica objetiva estructurada, los portafolios, las videograbaciones, los informes finales, etc. Estos instrumentos dan la oportunidad de ofrecer feedback sobre la actuación y su posterior reflexión. Especial consideración en la evaluación debería tener la observación directa de una actuación, ya que es el mejor método de evaluación de las competencias (de forma autónoma algunos estudiantes fueron haciendo anotaciones en las encuestas e historias clínicas, que matizaban algunos aspectos de las actividades formativas; para próximas convocatorias se propone elaborar un cuaderno o portafolio, en el cual los estudiantes puedan hacer esto de forma sistemática y estructurada). Los instrumentos de evaluación han de ser variados y amplios para garantizar la validez, fiabilidad, flexibilidad e imparcialidad [13,14].

\footnotetext{
Bibliografía

1. Espacio Europeo de Educación Superior. Real Decreto 55/2005, de 21 de enero, por el que se establece la estructura de las enseñanzas universitarias y se regulan los estudios universitarios oficiales de grado.

2. Programa de Medicina. Plan de estudios. Informe ANECA Grado de Medicina de la Universidad de Granada.
} 
3. Ponencia I: Convergencia Europea en el Grado. Educ Med 2005; 8: 113-4.

4. General Medical Council. Tomorrow's doctors: recommendations on undergraduate medical education. London: General Medical Council; 1993.

5. Cade J. An evaluation of early patient contact for medical students. Med Educ 1990; 3: 25-33.

6. Zabalza MA. El practicum y las prácticas en empresas en la coordenadas de la convergencia. Ponencia presentada en el VIII Simposio Internacional sobre el Practicum y las Prácticas en Empresas en la Formación Universitaria. Santiago: Poio; 2005.

7. Tejada J. El trabajo por competencias en el practicum: cómo organizarlo y cómo evaluarlo. Revista Electrónica de Investigación Educativa 2005. URL: http://redie.uabc.mx/ vo7no2/contenido-tejada.html. [20.02.2011].

8. Zabalza MA. Competencias docentes del profesorado universitario. Calidad y desarrollo profesional. Madrid: Narcea; 2003.
9. López MC, Romero MA. Estructura y recursos en el practicum de la licenciatura de pedagogía. Un estudio comparado. Revista Iberoamericana de Educación 2010; 53: 1-12.

10. Slick SK. The university supervisor: a disenfranchised outsider. Teach Teach Educ 1998; 14: 821-34.

11. Field B. Teachers as mentors: a practical guide. London: Falmer Press; 1994.

12. Molina-Ruiz E. El practicum de pedagogía en la Universidad de Granada: aspiraciones y realidad. In Raposo M, Cid A, Sanmamed M, Iglesias L, Muradas M, Zabalza MA, eds. El practicum en el nuevo contexto del Espacio Europeo de Educación Superior. Santiago: Imprenta Universitaria; 2005. p. 707-14.

13. McDonald, R, Boud D, Franci J, Gonczi A. Nuevas perspectivas sobre evaluación. Boletín Cinterfor 2000; 149: 41-72.

14. Universidad de Granada. Departamento de Medicina. Patología General. Prácticas. URL: http://wdb.ugr.es/ medicina/ pregrado.php?apartado $=7 \&$ asignatura $=5 \&$ titulacion $=1$. 